\title{
Understanding the Power of Hope and Meaning: Lessons Learned from Religion and Mysticism
}

\author{
Klostermann $\mathrm{K}^{1 *}$, Mignone $\mathrm{T}^{2}$, Peressotti $\mathbf{M}^{1}$, Jerard $\mathrm{A}^{1}$, Mahadeo \\ $\mathrm{M}^{1}$ and Papagni E 1 \\ ${ }^{1}$ Medaille College, 18 Agassiz Circle, Buffalo, NY, USA \\ 2VA Western New York Healthcare System, 3495 Bailey Avenue, Buffalo, NY, USA
}

\section{Review Article \\ Volume 3 Issue 3}

Received Date: February 26, 2018

Published Date: April 05, 2018

*Corresponding author: Keith Klostermann, Medaille College, 18 Agassiz Circle, Buffalo, NY, USA, Tel: 716-932-2559; Email: kck35@medaille.edu

\section{Abstract}

Studies have shown that people are more willing to seek out a mystic healer or rely on religion and spirituality for healing than engage in psychotherapy. Accordingly, it is important that mental health practitioners better understand the mechanisms by which religion and mysticism engage people as well as how they provide their clients with a sense of purpose and meaning. In doing so, psychological practitioners may benefit from identifying the ways these fields connect with their audience. The purpose of the current paper is to delineate the common threads or similar goals of mysticism, religion, and psychotherapy. In addition, the manuscript highlights divergent themes that may have crucial implications for clinical practice - specifically, take-home messages about what can be learned from these disciplines, what psychotherapy is missing, and how psychological practice might be enhanced. Recommendations are provided for the practicing clinician.

Keywords: Mysticism; Religion, Psychotherapy; Perception; Enhanced

\section{Introduction}

\section{Understanding the Power of Hope and Meaning: Lessons Learned from Religion and Mysticism}

Mysticism has been defined as "any belief in the existence of realities beyond perceptual or intellectual apprehension, or central to being and accessible to consciousness" [1]. More simply put, mysticism is experienced via our sensations and feelings; it cannot be explained by human intellect (Dean, 1969). Dean (1969) provides examples of mysticism, which include, but are not limited to: dreams, faith healing, and extrasensory perception (i.e., having second sight or sixth sense). In many ways mysticism possesses religious properties, but on the opposing side of the spectrum it may provide consumers with a means of entertainment as they inadvertently seek out new knowledge about themselves, those in their lives, and what their future holds. Mysticism may also provide individuals with immediate, concrete answers, the opposite of which is true in psychotherapy. For example, a psychotherapist's goals and plan may not 


\section{Psychology \& Psychological Research International Journal}

be immediately apparent to a client who is dealing with grief, eliciting frustration in said client. This client may yearn for closure directly from their loved one, which is not feasible through psychotherapy. Mysticism, on the other hand, can provide a means of communication between the client and their departed loved one; this may be exactly what the client needs. Notwithstanding the various reasons why one may turn to mysticism, we argue that there are lessons to be learned for the psychological practitioner offering psychotherapeutic services.

The purpose of this paper is to delineate the common threads or similar goals of mysticism, religion, and psychotherapy. In addition, this paper strives to highlight divergent themes that may have crucial implications for clinical practice - specifically, take-home messages about what can be learned from these disciplines and where psychotherapy may be lacking.

\section{The Purpose of Mysticism: What It Provides Believers}

Mysticism appears to be more than just traditional 'fortune-telling', 'palm-reading', and 'guessing cards' (Dean, 1976); for some, it may bring sacred meaning, relief, and/or can even serve as a survival function [1]. For example, consumers might seek out such services to become familiar with the unexpected and to find an inkling of certainty or predictability in ambiguity. Dean (1976) suggests that components of extrasensory perception (ESP), for which many mystic healers proclaim to possess, can act as a warning system or "psychic radar."By obtaining information that was once perceived to be unforeseeable, consumers can then prepare themselves adequately and appropriately in order to respond to imminent danger that proceeds them (Dean, 1976). In this way, mysticism (e.g. psychic healing) has the potential to not only warn but encourage consumers to think ahead about how they can stay strong, preserve their existence and well-being, and overcome what is yet to come. Supplementary to providing consumers with information about unanticipated danger, psychics, mediums, card readers, and so forth, possess "psychic radar" that also detects success and/or potential grand life achievements. Accordingly, levels of anxiety may dissipate, and self-confidence may be gained or increased.

\section{The Purpose of Religion: What it Provides Members}

Ascribing to a particular religious faith can infiltrate all aspects of an individual's life. Individuals' perceptions of the self, others, world, and future may be impacted by the entwinement of their religious faith and personal identity [2]. Organized religion provides members with a sense of security, as it creates a community to which all members belong [3]. This, in and of itself, can foster mental health as it provides an inherent social microcosm, eliciting feelings of universality and cohesion to give members the perception that they are not alone on life's journey [4]. Galek, Flannelly, Ellison, Stilton, and Jankowski (2014) articulate that organized religion also guides members in uncovering their own life-meaning. In doing so, it fosters a sense of self-worth in that members believe there is a purpose for their existence, even when facing difficult or challenging circumstances. Such purpose helps members feel as if their life is not finite, but rather that their good deeds will live on. This in turn creates a sense of value within the individual, motivating him or her to establish and accomplish meaningful goals, further perpetuating a sense of mastery and positive self-esteem. Abiding by religious rituals and teachings helps members feel as if their day-to-day actions are reasonable and justified [5]. Ultimately, religion can have profound implications for individuals, ranging from being a minor contributor in their daily life to being a central influence on their overall way of being [4].

\section{Common Threads: Mysticism, Religion and Psychotherapy}

Mysticism and psychotherapy share common ingredients, with overlap pertaining to techniques and desired outcomes. Akin to mysticism, which strives for heightened personal awareness, and for individuals to achieve "a wider, sharper, consciousness" about their existence that is "...more profound", psychotherapy fosters self-insight, self-understanding, and may focus on bringing one's attention to unconscious motivations to expand awareness [6].

Dreams have been continuously examined throughout mystic history. They have been used to increase one's contact with reality, god, one's own spiritual being or divine self through meditation, contemplation, and the use of self-affirmation mantras, journalism, imagery, and/or prayer and poetry [6]. In the same way, psychotherapists from diverse therapeutic schools employ such techniques. Thus, both the mystic and psychotherapist undertake remedial activities, whether it be interpreting dreams, re-examining painful memories, utilizing exercises that encourage concentration and relaxation, and/or practicing positive self-talk and guided visualizations. Such techniques can be thought of as renewed mystical traditions that are operationally pertinent for both disciplines. 


\section{Psychology \& Psychological Research International Journal}

Although there is an array of procedures that fall under the umbrella of mysticism (e.g. card reading, fortune telling, psychic healing, telepathy), we can still determine underlying commonalities for each which can also be applied to psychotherapy. Dean (1976) outlines 5 common denominators that are fundamental to all healing practices, supernatural or not. He purports that healing practices are: 1) self-reparative, 2) use the power of suggestion, 3) place emphasis on the reputation and therapeutic personality of the therapist or healer, 4) recognize the faith of the patient/client, and 5) believe in the transfer of energy from the healer (whomever this may be) to the patient/client. To this end, both disciplines contain restorative properties, place significance on the therapeutic relationship, and recognize the influence and expertise at the hands of the therapist/healer. Furthermore, both acknowledge client factors that drive the change process [1].

Mysticism and psychotherapy also share several common elements with organized religions. Converging with mysticism and psychotherapy, religion attempts to nurture increased insight. For example, meditation practices of the Buddhist faith have been adopted by several theoretical orientations within psychology as effective techniques to foster greater self-awareness, inner peace, and compassion [7]. Probing further, many religions discuss forgiveness in both scripture and doctrine. Menahem and Love (2013) articulate that to forgive, one must replace previous thoughts of anger and resentment with newfound empathetic thoughts. This is similar to the thought replacing technique within Cognitive-Behavioral Therapy (CBT), in which individuals are asked to identify and challenge maladaptive thoughts related to their psychopathology, allowing such thoughts to be replaced with healthier outlooks. A similar concept related to forgiveness is present in the psychodynamic model. In this model, individuals undergo narrative therapy to recreate and conceptualize one's life story in a more adaptive manner to alleviate prior resentments and self-blame. Ultimately, meditation and contemplation are mindfulness techniques that span mysticism, religion, and psychotherapy. They promote perspective taking and allow individuals to build greater awareness of self and compassion for others [7].

In addition to converging techniques, there are several similarities among the approach taken by mystics, spiritual healers, religious leaders, and psychotherapists. Similar to psychotherapists, religious leaders and spiritual healers often attempt to establish a sense of rapport before embarking on the treatment process [4].
While not explicitly stated as rapport building, Leighton and Leighton (2011) describe the religious practice of the Navaho Indians in which ill individuals are treated by a medicine man (also referred to as "Singer"). The Singer is responsible for conducting several extravagant rituals (e.g., ritual bath, sacrifices, sand paintings, special songs and prayers), wherein he cures the individual of his/her physical or mental ailments. Prior to ceremonial commencement, the Singer spends his first evening becoming acquainted with the ill patient and his/her family. This parallels the therapeutic rapport building of collaborative psychotherapy. A similar emphasis is also placed on the relationship between the healer and member within Vodou healing, further stressing the parallel between psychotherapy and religious healing $[3,4]$.

Other psychotherapeutic factors that can be deemed consistent with Vodou healing practices include: diagnostic interviewing, suggested or hypothesized case conceptualizations, and the elicitation of feedback from the ill member. Empathic listening is also central to both psychotherapy and Vodou healing. Congruent to clientcentered therapies, Vodou healers recognize the importance of obtaining the member's own postulated reason(s) for his/her suffering; placing agency at the hands of the ill member. In doing so, a collaborative treatment plan is constructed and agreed upon; this is also evident in psychotherapeutic practice.

\section{Diverging Themes among Mysticism, Religion, and Psychotherapy}

Despite the aforementioned similarities, mysticism, religion and psychotherapy also differ in several important ways. For example, psychologists diagnose based on specific criteria laid out by the Diagnostic and Statistical Manual of Mental Disorders, $5^{\text {th }}$ Edition (DSM5), whereas healers of different domains more often than not attribute psychological distress to a supernatural force [1]. The DSM-5 stems from an array of scientific and empirical research; psychometrically sound assessment protocols have been developed to objectively identify and categorize psychological distress, aiding clinicians in discerning the most accurate diagnosis. Conversely, many religions view mental health as a negative omen based on years of tradition and passed on teachings [3].

When members of certain religious groups fall ill to psychological distress, the community is frequently involved in ritualistic ceremonies to help guide the individual back to health. This stems from a collectivistic belief system in which the group is more important than 


\section{Psychology \& Psychological Research International Journal}

the individual on his/her own. On the other hand, it is commonplace within Western medicine to idealize independence and autonomy associated with individualism. Such an individualistic ideal is a driving force in Western society. In this way, mental health is regarded as an individual problem; a problem that needs to be figured out on one's own behalf, creating a greater atmosphere for potential stigma. As a result, when one is mentally-ill, they are more likely to seek out individual psychotherapy, whereas individuals from collectivistic cultures are more likely to pursue aid from the church, faith community, and government [4].

\section{Clinical Implications: What Psychotherapy can Gain from Mysticism and Religion}

"Religious experience is absolute; it cannot be disputed. You can only say that you have never had such an experience, whereupon your opponent will reply: Sorry, I have. And there your discussion will come to an end"Jung CJ [8].

But the conversation need not end, and topics surrounding religion, spirituality, and mysticism should not be avoided behind closed doors and while in session. Research has demonstrated that religion and spirituality can meaningfully impact clients' lives. Thus, these are important facets that need to be uncovered and addressed in psychotherapy [9]. We contend that psychotherapists can gain valuable knowledge regarding a client's beliefs by taking their clinical questioning beyond surface level exploration. Psychologists expect their clients to be forthcoming through personal disclosures; yet, all too often, psychologists are hesitant to express their own ignorance and lack of understanding with respect to clients' religious or spiritual beliefs. Psychologists must be able to take a step back and ask clients to help them understand what their beliefs mean to them, specifically at an individual level; this can aid in fostering an accurate and detailed conceptualization of the client and guards against injecting implicit biases into the treatment plan.

Clinical considerations should include the client's religious/spiritual/mystical beliefs pertaining to their existence, which likely play a chief role in their daily lives. Barnett (2016) highlights two reasons why this should take precedence, or at least not be overlooked. Firstly, clients may present to therapy as a result of religious and/or spiritual internal conflict; this may be the sole area of concern or may surface in time. For instance, clients may be experiencing distress due to faith crises such as: feelings of abandonment from God or the higher power for which they believe in, a life style and/or belief system that is incongruent with religious teachings, and/or thinking in extremes (i.e., misapplying the word of God or religious practices. Psychotherapists should also consider (or perhaps reconsider) discussions about religion, spirituality, and/or mysticism because these are probable areas of strength and support for clients. Accordingly, they should be regarded as such when appropriate, and accessed more frequently alongside ongoing treatment [9]. In aiding our clients in their reconnection to their faith communities, psychotherapy must integrate religious and spiritual interventions into the therapeutic endeavor.

Richards and Bergin (cited by West, 2000, n.p.) identify ways in which counselors can implement spirituality into their office; such interventions include:

"praying for clients, encouraging clients to pray, discussing theological concepts, making references to scriptures, using spiritual relaxation and imagery techniques, encouraging forgiveness, helping clients live congruently with their spiritual values, self-disclosing spiritual beliefs or experiences, consulting with religious leaders, and using religious bibliotherapy" $[10,11]$.

It is vital to note that implementation and integration of faith into therapeutic practice poses ethical issues and responsibilities on behalf of clinicians. Although multiculturalists and spirituality-centered approaches to psychotherapy are more sensitive to cultural differences among clientele, they still require underlying evidencebased treatment methodologies and competency. Barnett (2016, p. 7) exemplifies this by noting that it is not okay for clinicians to try out religious or spiritual interventions because they understand these strategies to work for themselves. Techniques should be rooted in research findings that show substantial evidence for effective improvement. Nevertheless, before integrating religion and spirituality into psychotherapy, a discussion should be had with the client about the how treatment will unfold. More specifically, the risks and benefits should be outlined, as well as the rationale for the recommended sequence of treatment. To help move this process forward, Barnett (2016) recommends the following [9]:

- Ask about religion and/or spiritual considerations at the outset of the relationship; specifically, during the intake session.

- Participate in ongoing workshops, training opportunities, or courses to build competency.

- Understand that religious and spiritual interventions fall along a continuum (Barnett, 2016, p. 8); thus, one 


\section{Psychology \& Psychological Research International Journal}

particular technique may work well for one client but not another, and the range of techniques utilized in session must be individualized for each client.

- Build community partnerships and maintain these relationships so that you can refer your clients for assistance when interventions are beyond your level of expertise.

- Consult, consult, consult.

Additive recommendations based on the literature review include:

1. Having knowledge of adaptive and maladaptive religious belief systems (i.e. belief systems that may exacerbate one's negative symptoms or interpretation of a situation), and to be able to distinguish these differences from one another. In having this area of competency, clinicians are more apt to intervene at a level that is beneficial for the client and to target unhealthy ways of perceiving and interpreting religious and spiritual doctrines that may be contributing to and exacerbating mental illness.

2. Clinicians should uphold the ethical standards of respect and beneficence by providing alternative treatment options that are in line with the client's culture. Furthermore, questions stemming from genuine curiosity and unconditional positive regard are more likely to be met with honesty while fostering a sense of safety and security within the therapeutic space.

3. Religion and mysticism provide followers/believers/members with a sense of meaning and existential purpose. As such, psychologists should put effort into helping clients find their own purpose within psychotherapy early on in the treatment process.

4. Regardless if a client is coming to therapy or seeking out another modality of healing, healers and clinicians alike should provide reassurance that clients are not "out of their mind" or "crazy" and commend them on their bravery and readiness to change.

\section{Conclusion}

Dean (1976) underscores that psychic healing is far more rampant and widespread than scientific medicine. While future research should follow up this claim, it is important that we gain knowledge about the purpose of religion and mysticism, identify similarities across religion/mysticism and psychotherapy, and contrast ways of how each discipline undertakes their prospective feat(s). In doing so, psychological practitioners can benefit from or receive added value by honing-in on what is absent or neglected from their field for which religion and mysticism draw people in [1].

Clinicians ought to be willing to ask questions about religion, spirituality and mysticism, so that treatment can be tailored to meet the needs of the client, and so that treatment does not go against belief system(s) and values that at a very basic level are interwoven and in tune with their precise being. In addition, spiritual resources should be incorporated and integrated into the treatment process, and in the same vein should be viewed as coping strategies; rather than "petty" or "nonsense". Overall, interventions need to be congruent with, and not disrespectful of the client's faith. Perhaps the greatest overarching similarity between mysticism, organized religion, and psychotherapy is that all provide a social system by which members are given support and guidance to navigate life's challenges in attempts at promoting mental health [4]. As such, if psychologists wish to expand their practice to diverse populations, they need to be willing to work synergistically with other spiritual domains including, but not limited to, religion and mysticism.

\section{References}

1. Dean SR (1976) Mental health and society: A quest for purpose in psychic research. New York, NY: S. Karger.

2. Johnson WB (2016) Confrontation of clinically salient religion in psychotherapy: Ethical considerations. Journal of Psychology and Christianity 35(4): 344356.

3. Leighton AH, Leighton DC (2011) Elements of psychotherapy in Navaho religion. 1941 Psychiatry 74(4): 283-292.

4. Méance G (2014) Vodou healing and psychotherapy. Routledge.

5. Galek K, Flannelly KJ, Ellison CG, Silton NR, Jankowski KRB (2015) Religion, meaning and purpose, and mental health. Psychology of Religion and Spirituality 7(1): 1-12.

6. Bullis RK, Div M (1992) Psychotherapists and the mystical process. Journal of Contemporary Psychotherapy 22(1): 43-49.

7. Menahem S, Love $M$ (2013) Forgiveness in psychotherapy: The key to healing: 


\section{Psychology \& Psychological Research International Journal}

Forgiveness. Journal of Clinical Psychology 69(8): 829-835.

8. Lines D (2006) Spirituality in counseling and psychotherapy. [Electronic resource]. London: Sage.

9. Barnett JE (2016) Are religion and spirituality of relevance in psychotherapy? Spirituality in Clinical Practice 3(1): 5-9.
10. Richards PS, Bergin AE (1997) A spiritual strategy for counseling and psychotherapy. Washington, DC: American Psychological Association.

11. West W (2000) Psychotherapy and spirituality: crossing the line between therapy and religion. [Electronic resource]. London: Sage, pp: 152. 